\title{
La Contribution de la Qualité dans l'Education Pour Tous En Afrique Subsaharienne: Une analyse basée sur l’approche Shapley-Owen-Shorrocks
}

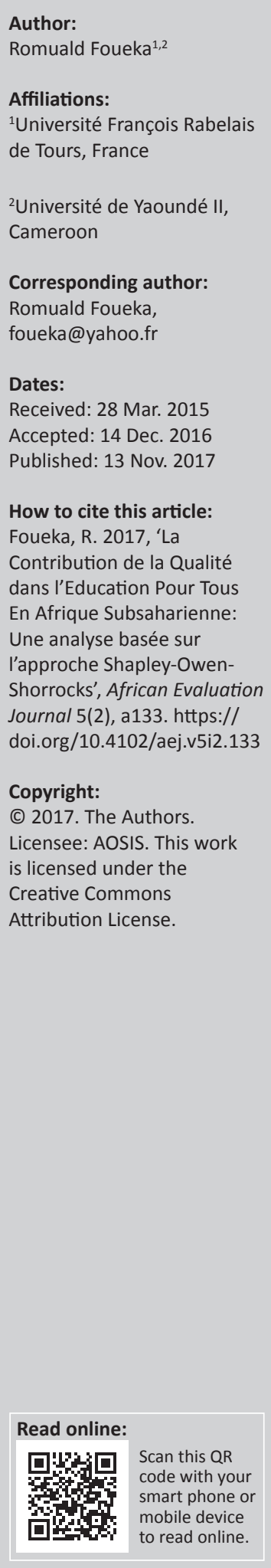

Contexte: Il est devenu évident que dans les pays africains, le droit à l'éducation ne devait pas se limiter au droit d'être admis à l'école. Dès lors, le défi d'améliorer l'éducation en Afrique subsaharienne comprenait non seulement une dimension quantitative mais aussi une dimension qualitative.

Objectif: Ainsi, notre étude avait pour objectif principal d'estimer la contribution de la qualité de l'éducation dans le développement de l'éducation pour tous (EPT) en Afrique francophone subsaharienne.

Méthode: A partir de l'IDE (Indice de Développement de l'EPT) développé par l'UNESCO, nous avons utilisé l'approche SHAPLEY-OWEN-SHORROCKS (SOS) pour apprécier l'impact de quatre composantes de cet indice : enseignement primaire universel, alphabétisation des adultes, parité entre les sexes, et en particulier la « qualité de l'éducation » qui semble de plus en plus agir très favorablement sur le développement économique dans les PED. Les données utilisées étaient les données secondaires compilées dans les rapports mondiaux sur le suivi de l'EPT produits chaque année par l'UNESCO depuis l'année 2002. Nous avons recueilli les données pour 12 pays d'Afrique francophone au Sud-Sahara.

Résultats: Nos résultats ont montré que, sur la période 1998-2011, les différentes composantes de l'IDE avaient des contributions diverses et variées. Par ordre décroissant de contribution à l'atteinte de l'objectif de scolarisation universelle on avait: le taux de scolarisation (49\%), l'indice relatif au Genre (21\%), le taux d'alphabétisation (20\%) et en dernière place la qualité de l'éducation (10\%).

Conclusion: Ainsi, ces résultats ont permis de conclure que la qualité de l'éducation contribuait très marginalement au développement humain dans divers pays africains.

The contribution of school quality in education for all in sub-Saharan Africa: An analysis based on the Shappley-Owen-Shorrocks approach

Background: It became clear that in African countries, the right to education should not be limited to the right to be admitted to school. From then, the challenge of improving education in sub-Saharan Africa has not only a quantitative dimension but also a qualitative dimension.

Objectives: Thus, our study's main objective was to estimate the contribution of the quality of education in the development of education for all (EFA) in sub-Saharan African countries.

Method: From the EDI (EFA Development Index) developed by UNESCO, we used the SHAPLEY-OWEN-SHORROCKS (SOS) approach to appreciate the impact of each component of this index: universal primary education, adult literacy, gender parity and especially the 'quality of education' component that seems increasingly act favourably on economic development in developing countries. The data used were secondary data compiled in the global reports on the monitoring of EFA produced each year since 2002. We collected data for 12 Francophone countries in Africa Sub-Sahara.

Results: Our results showed that, over the period 1998-2011, the different components of FDI had various and varied contributions. In descending order of contribution to the achievement of universal education objective, we have: the enrolment rate (49\%), the index on the Gender $(21 \%)$, the literacy rate $(20 \%)$ and last place the quality of education $(10 \%)$.

Conclusion: Thus, these results suggested that the quality of education marginally contributed to human development in various African countries. 


\section{Introduction}

Les bilans comparatifs effectués par l'UNESCO en 2004 soulignent le développement insuffisant et le mauvais fonctionnement de l'école en Afrique subsaharienne. C'est une grande préoccupation pour les gouvernements nationaux et les organisations internationales. Sans insister outre mesure sur les ressorts historiques d'une telle situation, il est important de réfléchir sur l'état actuel des systèmes scolaires, les évolutions qui se dessinent et les innovations que l'on peut observer pour trouver les termes dans lesquels se pose l'objectif spécifique de scolarisation universelle dans cette sous-région.

Depuis la Déclaration de Jomtien (1990) et surtout avec le cadre d'action de Dakar (2000), la communauté internationale reconnaît que la qualité de l'éducation est un déterminant primordial de la réalisation de L'Education Pour Tous (EPT) Il importe peu qu'un enfant soit scolarisé s'il arrête malgré tout ses études en raison de la qualité insuffisante de l'enseignement ou s'il achève un cycle d'enseignement sans savoir lire, écrire, compter, évaluer ses options ou prendre des décisions importantes dans la vie. Ainsi, il est de plus en plus admis que tous les systèmes éducatifs du monde doivent avoir pour objectif de transmettre aux enfants et aux adolescents des connaissances, des savoirs et une culture commune. Il est devenu évident que dans les pays africains, le droit à l'éducation ne doit pas se limiter au droit d'être admis à l'école. Dès lors, le défi d'améliorer l'éducation en Afrique subsaharienne comprend non seulement une dimension quantitative mais aussi une dimension qualitative.

En plus de leur absence souvent très remarquée dans les classements internationaux, la problématique de la qualité s'inscrit dans un contexte éducatif et économique plus que difficile dans certains pays. Dans ce palmarès des difficultés, les systèmes éducatifs des pays d'Afrique, et plus particulièrement ceux d'Afrique francophone sont, malheureusement, très bien placés. Ces pays s'engagent dans une bataille qui doit se mener simultanément sur deux fronts: celui de la quantité et celui de la qualité. Il faut, avec peu de moyens financiers dont ces pays disposent, scolariser davantage d'enfants dans une école où les enfants doivent apprendre suffisamment.

Au regard des différents rapports mondiaux sur le suivi de l'EPT on s'aperçoit que dans les pays francophones d'Afrique subsaharienne l'objectif d'EPT marque le pas depuis 1998 (voir tableau ci-dessous). L'UNESCO a développé l'indice du développement de l'éducation pour tous (IDE) qui est un indice composite qui permet d'obtenir une évaluation globale du système éducatif d'un pays par rapport aux objectifs de l'EPT. Les éléments constitutifs de l'IDE devraient dans l'idéal refléter la totalité des 6 objectifs, mais cela est difficile en pratique. L'objectif 3, relatif aux programmes d'apprentissage et à ceux concernant les compétences nécessaires dans la vie courante, n'est pas facile à quantifier, tandis que l'objectif 1 , relatif à la Protection et Education de la
Petite Enfance (PEPE), ne peut encore être incorporé car les données nationales sur les effectifs ne sont disponibles que pour quelques États et elles ne sont pas suffisamment standardisées. En conséquence, l'IDE standard ${ }^{1}$ est pour un pays donné la moyenne arithmétique des valeurs observées des indicateurs de : l'enseignement primaire universel (mesuré par le taux net ajusté de scolarisation dans le primaire), l'alphabétisation des adultes (mesurée par le taux d'alphabétisme des personnes âgées de 15 ans et plus), la parité entre les sexes (mesurée par l'indice de l'EPT relatif au genre qui est la moyenne des indices de parité entre les sexes des taux bruts de scolarisation et du taux d'alphabétisme des adultes) et la qualité de l'éducation (mesurée par le taux de survie en 5e année du primaire). La valeur de l'IDE se situe entre 0 et 1,1 représentant la pleine réalisation de l'éducation pour tous de chacun des quatre objectifs retenus. Le tableau ci-dessous illustre l'évolution de l'IDE pour certains pays francophones² au cours de la période 1998-2010 (Tableau 1).

Ce tableau illustre bien le retard accusé par les pays francophones d'Afrique subsaharienne dans le processus d'atteinte de l'EPT. Afin de permettre la définition des meilleures stratégies de relance, il est nécessaire de partager les responsabilités de ce retard en particulier la responsabilité de la qualité de l'éducation dans l'atteinte de 1'EPT. Ainsi, au regard des faibles performances enregistrées par les pays francophones au Sud du Sahara, notre étude se fixe pour objectif principal de voir quelle est la contribution de la qualité de l'éducation dans le développement de l'EPT en Afrique francophone. De façon spécifique, il s'agit de décomposer l'IDE et d'évaluer la contribution de chaque composante en particulier celle de la qualité de l'éducation.

\section{Revue de la littérature}

Il est bien établi que les avantages tirés de l'éducation par les individus et la société sont renforcés quand cette éducation est de qualité. Le rapport mondial sur le suivi de l'EPT, l'exigence de la qualité, s'attache principalement à faire ressortir l'impact de la qualité de l'éducation (compétences et acquisition des connaissances) sur : les revenus des individus, la croissance, les compétences non cognitives et les changements comportementaux. De plus en plus d'éléments prouvent que la qualité des ressources humaines est directement liée aux revenus individuels, à la productivité et à la croissance économique (Hanushek et Wossmann 2007:11). Autrement dit, à durée de scolarité égale, les résultats d'une meilleure qualité de l'éducation sont étroitement liés aux différentiels ultérieurs de revenus et donc, aux différences de productivité individuelle. En conséquence, les programmes éducatifs qui inculquent ces compétences apportent plus de bénéfices économiques individuels que ceux qui ne le font pas.

1.L'IDE élargi incorpore, en plus des éléments de I'IDE standard, I'indicateur de l'objectif $1 . \mid l$ est calculé dans le rapport 2012 pour les pays disposant de cette statistique. Ce qui n'est pas le cas pour la majorité des pays que nous avons considérés dans cette étude.

2.Le Mali, le Burkina Faso et le Niger occupent aussi bien en 2010 qu'en 1999 les trois dernières places des classements mondiaux. 
TABLEAU 1: Evolutionde l'IDE et de ses composantes entre 1998 et 2010.

\begin{tabular}{|c|c|c|c|c|c|c|c|c|c|c|c|}
\hline \multirow{2}{*}{\multicolumn{2}{|c|}{$\begin{array}{l}\text { Pays } \\
\text { (classement 2010) }\end{array}$}} & \multicolumn{2}{|c|}{ IDE } & \multicolumn{2}{|c|}{ TNS } & \multicolumn{2}{|c|}{ TAA } & \multicolumn{2}{|c|}{ IEG } & \multicolumn{2}{|c|}{ TS } \\
\hline & & \multirow{2}{*}{$\begin{array}{c}1998 \\
0.709 *\end{array}$} & \multirow{2}{*}{$\begin{array}{c}2010 \\
0.781\end{array}$} & \multirow{2}{*}{$\begin{array}{c}1998 \\
0.840^{*}\end{array}$} & \multirow{2}{*}{$\begin{array}{c}2010 \\
0.987\end{array}$} & \multirow{2}{*}{$\begin{array}{c}1998 \\
0.692^{*}\end{array}$} & \multirow{2}{*}{$\begin{array}{l}2010 \\
0.711\end{array}$} & \multirow{2}{*}{$\frac{1998}{0.904^{*}}$} & \multirow{2}{*}{$\begin{array}{c}2010 \\
0.956\end{array}$} & \multirow{2}{*}{$\begin{array}{c}1998 \\
0.400 *\end{array}$} & \multirow{2}{*}{$\begin{array}{l}2010 \\
0.472\end{array}$} \\
\hline Rwanda & $103^{e}$ & & & & & & & & & & \\
\hline Togo & $106^{e}$ & 0.633 & 0.742 & 0.898 & 0.943 & 0.544 & 0.571 & 0.575 & 0.679 & 0.516 & 0.777 \\
\hline Mauritanie & $107^{e}$ & 0.605 & 0.732 & 0.627 & 0.744 & 0.392 & 0.580 & 0.749 & 0.862 & 0.652 & 0.743 \\
\hline Sénégal & $109^{e}$ & 0.587 & 0.707 & 0.579 & 0.780 & 0.356 & 0.497 & 0.646 & 0.815 & 0.767 & 0.737 \\
\hline Mali & $118^{e}$ & 0.492 & 0.612 & 0.386 & 0.620 & 0.241 & 0.311 & 0.558 & 0.678 & 0.783 & 0.840 \\
\hline Niger & $120^{e}$ & 0.393 & 0.528 & 0.261 & 0.572 & 0.150 & 0.287 & 0.547 & 0.612 & 0.613 & 0.643 \\
\hline
\end{tabular}

Source: Rapports mondiaux sur le suivi de l'EPT 2012 et 2006

Les valeurs en gras sont les valeurs de l'IDE en 1998 et 2010. Les valeurs de l'IDE et de ses composantes sont comprises entre 0 et 1 . Plus le chiffre se rapproche de 1 , plus on tend vers l'atteinte de l'objectif.

*, ces données correspondent aux statistiques de l'année 2001 pour le Rwanda.

IDE, Indice de Développement de l'EPT; TNS, Taux Net de Scolarisation; TAA, Taux d'Alphabétisation des Adultes; IEG; Indice relatif au Genre; TS, Taux Survie en 5 e année.

Bien que les données sur ces relations soient quelque peu limitées dans les PED, il semble probable que les bénéfices résultant de la qualité de l'éducation y soient plutôt plus élevés que dans les pays industrialisés. Utilisant des mesures simples des compétences cognitives de base, des études de six pays en développement (Ghana, Kenya, Maroc, Pakistan, Afrique du sud et Tanzanie), citées dans Hanushek (2005:17), montrent que celles-ci génèrent des bénéfices économiques importants (abstraction faite de l'effet que peut avoir le nombre d'années de scolarité). C'est uniquement dans les cas portant sur le Ghana et la Tanzanie qu'on trouve une fourchette de bénéfices inférieure ou comparable à celle des estimations des États-Unis. Dans les quatre autres pays, une augmentation d'un point d'écart type des aspects qualitatifs de l'éducation était associée à un accroissement des salaires de 12 à $48 \%$, ce qui témoigne d'un rendement significatif de l'élévation des niveaux de compétences cognitives et donc, probablement, de l'amélioration de la qualité de l'éducation (Hanushek 2005:16). Des recherches empiriques ${ }^{3}$ ont aussi démontré qu'une éducation de qualité améliore le potentiel économique national. La qualité de la main-d'œuvre, là encore mesurée par les scores aux tests, semble être un déterminant important de la croissance économique et, donc, de l'aptitude des gouvernements à réduire la pauvreté.

Une partie des bénéfices associés à la qualité de l'éducation viennent également de la poursuite de la scolarité qui augmente l'acquisition des connaissances et des compétences et réduit les coûts éducatifs du fait de la diminution des taux de redoublement et d'abandon. L'amélioration des acquis cognitifs est étroitement liée aux chances de fréquenter l'école plus longtemps. Ainsi, les écoles et les systèmes scolaires de meilleure qualité tendent à présenter des taux de survie plus élevés.

A l'exception de l'étude de Saha (2005), les études sur la contribution de la qualité de l'éducation sur le développement humain font toujours défaut dans les PED, particulièrement ceux d'Afrique subsaharienne. Mais le développement par l'UNESCO depuis 2005 de l'IDE, nous permet à travers les diverses techniques de décomposition, d'apprécier dans les

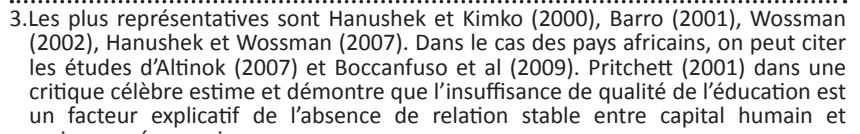
critique célèbre estime et démontre que l'insuffisance de qualité de l'éducation est un facteur explicatif de l'absence de relation stable entre capital humain et croissance économique. pays d'Afrique subsaharienne la contribution de chaque composante de l'indice dans l'atteinte de l'objectif de scolarisation universelle.

Afin de surmonter les difficultés auxquelles les économistes se heurtent lorsqu'ils proposent un arbitrage entre la logique économique, basée sur le calcul de la contribution d'un facteur à l'inégalité totale, et la logique mathématique, permettant de légitimer une mesure, Auvray et Trannoy (1992:7) proposent une généralisation de la technique de décomposition en facteurs par l'utilisation de la valeur de Shapley. Cette idée est reprise par Chantreuil et Trannoy (1997:90), Sastre et Trannoy (2002:55) et par Shorrocks (1999:7). Ce dernier retrouve à ce propos son résultat de 1982 concernant les mesures de la variance et du coefficient de variation au carré et démontre en faisant recours aux travaux d'Owen (1977:82) que la valeur de Shapley peut aussi être appliquée dans d'autres domaines, comme celui des mesures de pauvreté. Cette démarche est désignée par l'approche SHAPLEY-OWEN-SHORROCKS (SOS) depuis les travaux de Shorrocks et Kolenilov (2001:12). Saha (2004:32) souligne que l'avantage de cette approche est qu'elle ne laisse aucun résidu et les travaux de Silber (2004:820) et d'autres ont confirmé que la valeur de Shapley est une très bonne technique de décomposition. Elle a été utilisée par plusieurs auteurs, notamment par Kabore (2002:17) et par Baye (2004:9) pour décomposer les variations de l'incidence de pauvreté respectivement au Burkina-Faso et au Cameroun et par Saha (2005:32) pour décomposer les variations de l'IDH dans les pays africains. La majorité de ces études décompose les indices ayant généralement deux facteurs, mais nous utilisons cette approche SOS pour décomposer un indice à quatre facteurs. Dans la suite, nous présentons le cadre théorique de l'approche, le mode d'application sur l'IDE et les données à utiliser.

\section{Méthodologie Cadre théorique de l'approche SOS}

La valeur de Shapley (1953:3), concept issu de la géométrie des simplexes, est utilisée en théorie des jeux coopératifs où l'on cherche à déterminer les modalités de partage d'une somme (un bénéfice, un coût, etc.). On suppose qu'un indicateur I est fonction de $\mathrm{q}$ facteurs 1 tels que : $1=1,2, \ldots, \mathrm{q}$ ). L'algorithme de Shapley permet de mesurer la contribution 
de chaque facteur 1 à la mesure de l'indicateur total I en supposant que ce facteur peut être éliminé lorsqu'on mesure I. Cet algorithme permet aussi de tenir compte de toutes les interactions de ce facteur avec les autres. L'indicateur I dépend donc d'un ensemble mouvant $R$. Il admet ainsi plusieurs formes fonctionnelles $\mathrm{V}(\mathrm{R})$ définies par :

$\mathrm{V}:\{\mathrm{R}: \mathrm{R} \subseteq \mathrm{L}\} \rightarrow \mathrm{R}$

Si on considère que le facteur 1 a été supprimé de l'ensemble des variables $L$, le nombre de facteurs restants est égal à $r$, le cardinal de R. En mesurant la différence entre l'indicateur global (sur l'ensemble de toutes les variables) et l'indicateur après la perte d'une variable 1 , on définit un impact marginal particulier associé à la variable l. L'opération est réitérée en supprimant 1 avec d'autres sources de revenu. La mesure de I permet alors de déterminer un nouvel impact marginal. On répertorie ainsi au total $\mathrm{q}$ ! impacts marginaux pour chaque source de revenu. Pour mesurer la contribution $C^{1}$ de chaque facteur 1 à l'indicateur totale, on calcule la moyenne pondérée :

$$
C^{l}(L, V)=\sum_{r=0}^{q-1} \sum_{R \subseteq L-\{1\}} \frac{r !(q-s-1) !}{q !} \Delta_{l} V(R)(1)
$$

Où $\left.\Delta_{l} V(\mathrm{R})=V(R \cup\{l\})-V(R)\right]$

$\Delta_{l} V(R)$ est un des impacts marginaux de la variable 1. Chantreuil et Trannoy (1997:96) montrent que la valeur Shapley est une règle de décomposition cohérente car la somme de toutes les contributions procure la mesure totale de l'inégalité :

$$
\sum_{l=1}^{q} C^{l}=I
$$

\section{Mode d'applicationàl'IDE}

Dans l'application de l'approche SOS à la décomposition du niveau ou des variations d'un indicateur comme l'indice de pauvreté, d'inégalité, de développement humain ou de développement de l'éducation, nous parlerons de $\mathrm{m}$ facteurs et non de $\mathrm{m}$ joueurs. Comme on l'a relevé ci-dessus, la littérature a déjà enregistré des applications sur des indices à deux facteurs $(m=2)$, généralement aisées (Baye 2004; Chantreuil et Trannoy 1997; Kabore 2002; Saha 2005). Peu d'études ont appliqué cette approche sur des indices de plus de deux facteurs à l'instar de Chameni et Miamo (2012:151) dans le cas des inégalités au Cameroun. L'IDE est théoriquement fonction de quatre facteurs (l'enseignement primaire universel, l'alphabétisation des adultes, la parité entre les sexes et l'égalité des sexes, et la qualité de l'éducation). On a:

$$
I D E_{t}=f\left(T N S_{t,} T A A_{t,} I E G_{t,} T S_{t}\right)
$$

Avec:

- TNS $_{\mathbf{t}}$ représente le taux net ajusté de scolarisation dans le primaire de l'année t;
- TAA $_{t}$ mesure le taux d'alphabétisme des personnes âgées de 15 ans et plus;

- IEG est l'indice de l'EPT relatif au genre qui est la moyenne des indices de parité entre les sexes des taux bruts de scolarisation, dans le primaire et dans le secondaire, et du taux d'alphabétisme des adultes;

- TS $_{t}$ correspond au taux de survie en 5e année du primaire qui permet selon l'UNESCO d'appréhender le niveau de la qualité de l'éducation d'un pays à l'année t.

On peut écrire toute variation de l'IDE pour un pays à l'année $\mathrm{t}$ comme il suit :

$$
\Delta I D E_{t}=I D E_{t}-I D E_{t-1}=f\left(\Delta T N S_{t} \Delta T A A_{t} \Delta I E G_{t} \Delta T S_{t}\right)
$$

Où, $\Delta X_{t}$ désigne les variations de l'indice et des quatre indicateurs des composantes de l'indice.

Pour faciliter l'application de la formule (1), on peut construire l'expression de la contribution de l'unique facteur qui nous intéresse et qu'on généralisera aux autres facteurs. Ainsi, sachant que $\mathrm{m}=4$, la formule littéralement devient:

$$
\begin{aligned}
C^{T S} & =1 / 4[\mathrm{~V}(\mathrm{TNS}, \mathrm{TAA}, \mathrm{IEG})-V(\varnothing)] \\
& +1 / 12[\mathrm{~V}(\mathrm{TNS}, \mathrm{TAA})-V(\varnothing)] \\
& +1 / 12[\mathrm{~V}(\mathrm{TNS}, \mathrm{IEG})-V(\varnothing)]+1 / 12[\mathrm{~V}(\mathrm{IEG}, \mathrm{TAA})-V(\varnothing)] \\
& +1 / 12[\mathrm{~V}(\mathrm{TNS}, \mathrm{TAA})-V(\varnothing \cup\{\mathrm{IEG}\})] \\
& +1 / 12[\mathrm{~V}(\mathrm{TNS}, \mathrm{IEG})-V(\varnothing \cup\{\mathrm{TAA}\})] \\
& +1 / 12[\mathrm{~V}(\mathrm{TAA}, \mathrm{IEG})-V(\varnothing \cup\{\mathrm{TNS}\})] \\
& +1 / 24[\mathrm{~V}(\mathrm{TNS})-V(\varnothing)]+1 / 24[\mathrm{~V}(\mathrm{TAA})-V(\varnothing)] \\
& +1 / 24[\mathrm{~V}(\mathrm{IEG})-V(\varnothing)] \\
& +1 / 24[\mathrm{~V}(\mathrm{TNS})-V(\varnothing \cup\{\mathrm{IEG}, \mathrm{TAA}\})] \\
& +1 / 24[\mathrm{~V}(\mathrm{TAA})-V(\varnothing \cup\{\mathrm{TNS}, \mathrm{IEG}\})] \\
& +1 / 2[\mathrm{~V}(\mathrm{IEG})-V(\varnothing \cup\{\mathrm{TNS}, \mathrm{TAA}\})]
\end{aligned}
$$

On lit:

- $C^{\mathrm{TS}}$ la contribution du facteur TS à l'indicateur global IDE ;

- $\quad V(\varnothing)=$ valeur de l'IDE en $\mathrm{t}$ si aucun facteur n'avait changé, c'est-à-dire si tous les quatre composantes avaient gardé leurs niveaux de l'année $\mathrm{t}-1$;

- $V(\varnothing \grave{E}\{T N S\})=V(T N S)$ est la Valeur de l'IDE en $t$ si seul le facteur TNS avait été considéré à son niveau courant, tous les autres ont gardé en $t$ leurs niveaux de l'année $t-1$.

\section{Données}

En vu de réaliser cette étude, les données utilisées sont les données secondaires qui proviennent essentiellement des différentes versions de données compilées dans les rapports mondiaux sur le suivi de l'EPT produits chaque année 
depuis l'année 2002. Le rapport 2014 présente les progrès accomplis dans 205 pays pour l'année 2011, mais l'IDE a été calculé pour 115 pays disposant de données relatives aux quatre indicateurs nécessaires pour calculer cet indice. La compilation des données annuelles issues de chaque rapport nous permet d'avoir une série statistique sur la période 19982011 qui constituera notre période d'étude. Cette compilation nous permet d'obtenir les données pour 12 pays d'Afrique francophone au Sud-Sahara. Ces pays se situent en majorité en Afrique occidentale (Mauritanie, Sénégal, Mali, Niger, Burkina Faso, Togo, Benin et Guinée $)^{4}$ et le reste constitue le contingent des pays d'Afrique centrale ${ }^{5}$ (Burundi, Cameroun, Djibouti, RCA, et Rwanda).

\section{Présentation et analyse des résultats}

Nous commençons la présentation détaillée des résultats de la décomposition pour un pays puis nous généralisons sur l'ensemble des pays afin d'en tirer les conclusions.

Le calcul de l'IDE par 1'UNESCO est fait pour le Cameroun ${ }^{6}$ dans les rapports de l'EPT 2012 et 2014. On y retrouve les valeurs de l'IDE du Cameroun pour les années 2008, 2010 et 2011. Le Cameroun est classé parmi les pays à faible ${ }^{7}$ IDE en 2008 et 2011 et parmi les pays à IDE moyen en 2010. La valeur de son IDE se situe respectivement à $0.77375,0.81$ et 0.79475 (voir tableau 2 ci-dessous) entre 2008 et 2011. Bien qu'occupant une place de choix en comparaison des autres pays membres du PASEC, le Cameroun est marqué par une évolution faible de son IDE (soit 0.0215 ) et peine encore à réaliser l'objectif d'EPT.

Les résultats de la décomposition de l'IDE, par application de l'approche SOS, permettent de voir qu'au Cameroun chaque composante de l'IDE participe différemment à l'objectif de scolarisation universelle. Ainsi, par ordre de grandeur, on voit que les actions en faveur d'une éducation de qualité favorisent davantage l'atteinte de l'EPT au Cameroun (64\%). Viennent ensuite les sous-objectifs de scolarisation primaire universelle $(60 \%)$ et de parité entre sexe (6\%). Par contre, les actions en faveur de l'alphabétisation des adultes sont porteuses d'un effet néfaste sur l'atteinte de l'EPT. La baisse du niveau d'alphabétisation au Cameroun a entrainé une baisse de l'IDE de l'ordre de 35\%.

Afin de valider ces résultats de la décomposition de l'IDE, nous avons étendu notre échantillon sur d'autres pays d'Afrique sub-saharienne ayant un système d'éducation francophone et disposant de données sur la période 1998-2011. Les résultats globaux sont présentés dans le tableau ci-dessous.

4.Les données pour la Cote d'ivoire et le Nigeria ne sont présentées que dans le rapport EPT 2014. Dès lors, ces deux pays ne peuvent figurer dans notre analyse.

5.Pour le Cameroun et la RCA, les données doivent encore être complétées pour les années 2001 à 2007.

6.Ce pays possède la valeur de l'IDE la plus élevée en 2011 parmi les pays étudiés.

7.Les pays sont classés en trois catégories en fonction de la valeur de I'IDE : la catégorie d'IDE supérieur (IDE $>0.95)$; la catégorie d'IDE intermédiaire $(0.80<$ IDE $<0.95)$; et la catégorie d'IDE bas (IDE $<0.80$ ).
TABLEAU 2: Evolution de l'indice de développement de l'EPT au Cameroun.

\begin{tabular}{|c|c|c|c|c|}
\hline Variable & 2008 & 2010 & 2011 & $\begin{array}{c}\text { Variation de la } \\
\text { période }\end{array}$ \\
\hline IDE & 0.77375 & 0.81 & 0.79475 & \\
\hline Variation & & 0.03625 & 0.01525 & 0.0215 \\
\hline TNS & 0.883 & 0.939 & 0.935 & \\
\hline Contribution & & 0.014 & -0.001 & 0.013 \\
\hline$\%$ contribution & & 38 & 7 & 60 \\
\hline TAA & 0.759 & 0.707 & 0.713 & \\
\hline Contribution & & -0.013 & 0.0015 & -0.0115 \\
\hline$\%$ contribution & & -35 & -10 & -53 \\
\hline IEG & 0.822 & 0.831 & 0.847 & \\
\hline Contribution & & 0.00225 & 0.004 & 0.00625 \\
\hline$\%$ contribution & & 6 & -26 & 29 \\
\hline TS & 0.629 & 0.763 & 0.684 & \\
\hline Contribution & & 0.0335 & -0.01975 & 0.01375 \\
\hline$\%$ contribution & & 91 & 129 & 64 \\
\hline Total $\%$ contribution & - & 100 & 100 & 100 \\
\hline
\end{tabular}

Source: Calculs de l'auteur

Les valeurs en gras indiquent la variation de l'IDE $\left(I^{2} E_{t}-I_{D E}\right)$ et celle de ses composantes. Les valeurs en italiques (en pourcentage et en valeur relative) représentent la part de variation de chaque composante de l'IDE sur la variation globale de l'IDE.

IDE, Indice de Développement de I'EPT; TNS, Taux Net de Scolarisation; TAA, Taux d'Alphabétisation des Adultes; IEG; Indice relatif au Genre; TS, Taux Survie en $5^{e}$ année.

Il en ressort que de 1998 à 2011, l'IDE pour les pays africains retenus s'est accru approximativement de 0.0966. Le taux de scolarisation a contribué pour 0.04709 à cet accroissement (soit une contribution de 49\%), l'alphabétisation participe pour $0.01951(20 \%)$, l'aspect genre y contribue à hauteur de $0.02025(21 \%)$ et la contribution de la qualité de l'éducation est en moyenne de $0.00981(10 \%)$ au cours de la période. Nous voyons bien que par ordre d'importance, c'est la massification de la scolarisation qui l'emporte dans les progrès en matière d'EPT et que les aspects qualitatifs sont relégués au dernier rang. Toutefois, cette conclusion tirée des valeurs de la décomposition moyenne inter-pays de l'IDE cache de nombreuses spécificités individuelles. Nous allons commencer par présenter quelques traits marquants de la décomposition de l'IDE par pays en s'appuyant sur « trois composantes » (la scolarisation, l'alphabétisation et le genre). L'analyse des aspects éducatifs liés à la qualité sera abordée séparément.

L'évolution de l'IDE est plus marquée au Niger où la variation globale de l'IDE s'élève à 0.17. L'IDE du Niger est passé de 0.393 en 1998 à 0.563 en 2011. Cette tendance est soutenue par les efforts enregistrés dans l'ensemble des indicateurs. On note que les niveaux de scolarisation et d'alphabétisation ont doublé au cours de la période. Pour la scolarisation, son taux est passé de $26 \%$ en 1998 à 64\% en 2011 et le taux d'alphabétisation de $15 \%$ à $29 \%$ sur la même période. L'objectif de parité « entre les sexes » a connu une faible évolution dans ce pays passant de $55 \%$ à $61 \%$. Ainsi, les contributions de chaque composante à la forte hausse de l'IDE sont de l'ordre $55 \%$ pour la scolarisation, $20 \%$ pour l'alphabétisation et $10 \%$ pour le genre. Malgré ce bon résultat, le Niger occupe toujours la dernière place au regard de la valeur brute de son IDE parmi les pays recensés et dans le classement mondial du dernier rapport EPT. A l'opposé du Niger, se trouve la Guinée qui est le seul pays ayant enregistré une baisse de son IDE au cours de la période 20042011. Cette baisse a été occasionnée par la forte chute de la qualité de l'éducation dans ce pays ainsi que par le niveau 
TABLEAU 3: Décomposition des variations de l'IDE dans plusieurs pays africains selon l'approche SOS sur la période 1998-2011.

\begin{tabular}{|c|c|c|c|c|c|c|}
\hline \multirow[t]{2}{*}{ Pays } & \multirow[t]{2}{*}{ Périoded'étude } & \multirow{2}{*}{$\begin{array}{c}\text { Variation globale } \\
\text { de I'IDE }\end{array}$} & \multicolumn{4}{|c|}{ Contribution } \\
\hline & & & TNS (\%) & TAA (\%) & IEG (\%) & TS (\%) \\
\hline Bénin & $2001-2008$ & 0.05275 & $\begin{array}{c}0.05375 \\
(102)\end{array}$ & $\begin{array}{c}0.00250 \\
(5)\end{array}$ & $\begin{array}{c}0.02775 \\
(52)\end{array}$ & $\begin{array}{c}-0.03125 \\
(-59)\end{array}$ \\
\hline Burkina Faso & $1998-2011$ & 0.15375 & $\begin{array}{c}0.06975 \\
(45)\end{array}$ & $\begin{array}{c}0.00950 \\
(6)\end{array}$ & $\begin{array}{c}0.05175 \\
(34)\end{array}$ & $\begin{array}{c}0.02275 \\
(15)\end{array}$ \\
\hline Burundi & $1998-2008$ & 0.14325 & $\begin{array}{c}0.14525 \\
(101)\end{array}$ & $\begin{array}{c}0.04875 \\
(34)\end{array}$ & $\begin{array}{c}0.00875 \\
(6)\end{array}$ & $\begin{array}{c}-0.05950 \\
(-42)\end{array}$ \\
\hline Cameroun & $2008-2011$ & 0.0215 & $\begin{array}{c}0.01300 \\
(60)\end{array}$ & $\begin{array}{c}-0.01150 \\
(-53)\end{array}$ & $\begin{array}{c}0.00625 \\
(29)\end{array}$ & $\begin{array}{c}0.01375 \\
(64)\end{array}$ \\
\hline $\begin{array}{l}\text { République } \\
\text { centrafricaine }\end{array}$ & $2008-2011$ & 0.01875 & $\begin{array}{c}0.00500 \\
(27)\end{array}$ & $\begin{array}{c}0.00500 \\
(27)\end{array}$ & $\begin{array}{c}0.00400 \\
(21)\end{array}$ & $\begin{array}{c}0.00475 \\
(25)\end{array}$ \\
\hline Djibouti & 1998-2008 & 0.11225 & $\begin{array}{l}0.06150 \\
(55)\end{array}$ & $\begin{array}{c}0.00775 \\
(7)\end{array}$ & $\begin{array}{c}0.01750 \\
(15)\end{array}$ & $\begin{array}{c}0.02550 \\
(23)\end{array}$ \\
\hline Guinée & 2004-2011 & -0.00725 & $\begin{array}{c}0.05000 \\
(-690)\end{array}$ & $\begin{array}{c}-0.01050 \\
(145)\end{array}$ & $\begin{array}{c}0.00950 \\
(-131)\end{array}$ & $\begin{array}{c}-0.05625 \\
(776)\end{array}$ \\
\hline Mali & 1998-2011 & 0.15875 & $\begin{array}{l}0.07150 \\
(45)\end{array}$ & $\begin{array}{c}0.02325 \\
(15)\end{array}$ & $\begin{array}{c}0.04075 \\
(25)\end{array}$ & $\begin{array}{c}0.02325 \\
(15)\end{array}$ \\
\hline Mauritanie & $1998-2011$ & 0.1495 & $\begin{array}{c}0.03150 \\
(21)\end{array}$ & $\begin{array}{c}0.04850 \\
(32)\end{array}$ & $\begin{array}{c}0.02775 \\
(19)\end{array}$ & $\begin{array}{c}0.04175 \\
(28)\end{array}$ \\
\hline Niger & $1998-2011$ & 0.17 & $\begin{array}{c}0.0940 \\
(55)\end{array}$ & $\begin{array}{c}0.03425 \\
(20)\end{array}$ & $\begin{array}{c}0.01625 \\
(10)\end{array}$ & $\begin{array}{c}0.02550 \\
(15)\end{array}$ \\
\hline Rwanda & 2001-2011 & 0.05325 & $\begin{array}{c}0.0180 \\
(34)\end{array}$ & $\begin{array}{c}0.03675 \\
(69)\end{array}$ & $\begin{array}{c}-0.00825 \\
(-15)\end{array}$ & $\begin{array}{c}0.00675 \\
(12)\end{array}$ \\
\hline Sénégal & $1998-2011$ & 0.12075 & $\begin{array}{c}-0.01225 \\
(10)\end{array}$ & $\begin{array}{c}0.05250 \\
(44)\end{array}$ & $\begin{array}{c}0.03525 \\
(29)\end{array}$ & $\begin{array}{c}0.04525 \\
(37)\end{array}$ \\
\hline Togo & $1998-2010$ & 0.10925 & $\begin{array}{c}0.01125 \\
(10)\end{array}$ & $\begin{array}{c}0.00675 \\
(6)\end{array}$ & $\begin{array}{c}0.02600 \\
(24)\end{array}$ & $\begin{array}{c}0.06525 \\
(60)\end{array}$ \\
\hline Moyenne inter-pays & - & 0.0966 & $\begin{array}{c}0.04709 \\
(49)\end{array}$ & $\begin{array}{c}0.01951 \\
(20)\end{array}$ & $\begin{array}{c}0.02025 \\
(21)\end{array}$ & $\begin{array}{c}0.00981 \\
(10)\end{array}$ \\
\hline
\end{tabular}

Source: Calculs de l'auteur

Les valeurs en pourcentage représentent la part de variation de chaque composante de l'IDE sur la variation globale de l'IDE. Les autres valeurs décimales indiquent la variation de l'IDE et celle de ses composantes.

IDE, Indice de Développement de l'EPT; TNS, Taux Net de Scolarisation; TAA, Taux d'Alphabétisation des Adultes; IEG; Indice relatif au Genre; TS, Taux Survie en $5^{e}$ année.

faible d'alphabétisation qui a malheureusement aussi diminué sur la période. Par contre, les composantes de scolarisation et de genre favorisent le développement de l'EPT dans ce pays (voir tableau 3).

Au regard du palmarès affiché sur la variation de niveau pour chaque composante, on s'aperçoit qu'en ce qui concerne la scolarisation et l'alphabétisation, le Burundi est le pays ayant fait le plus de progrès. Le secteur de l'éducation au Burkina Faso a, quant à lui, connu la meilleure amélioration sur la parité fille/garçon. Cette hiérarchie est totalement bouleversée lorsqu'on tient compte des contributions individuelles. Ainsi, les améliorations du niveau de scolarisation et du taux de parité en Guinée ont contribué le plus à l'avancée de l'EPT dans ce pays et ce de façon relative aux autres contributions observées dans les mêmes composantes des autres pays retenus. Cette contribution positive est toutefois faible par rapport aux contributions négatives des autres composantes qui ont constitué des obstacles à l'atteinte de l'EPT dans ce pays. En matière d'alphabétisation, c'est l'augmentation observée au Rwanda qui possède la plus grande contribution.

Globalement on peut faire un classement de deux groupes de pays : un premier groupe où la qualité de l'éducation impacte positivement dans les progrès en matière $\mathrm{d}^{\prime} \mathrm{EPT}$ et un second groupe où cet impact est négatif. Le premier groupe regorge plus de pays à savoir : Cameroun, RCA, Burkina Faso, Djibouti, Mali, Mauritanie, Niger, Sénégal, Rwanda et Togo. Le second groupe par contre n'est constitué que de trois pays dont le Bénin, le Burundi et la Guinée. Quelle que soit l'appartenance d'un pays à un groupe quelconque, la contribution de la qualité de l'éducation varie profondément, ce qui impose un regard attentif sur ce sous-objectif dont l'impact sur le développement s'est avéré considérable.

Parmi les pays disposant d'une base de données plus large (1998-2011), le Togo est celui où la variation du niveau de qualité de l'éducation contribue le plus significativement au progrès du développement de l'EPT. Mais aussi, en termes de contribution relative, c'est au Togo que l'on observe un taux considérable $(60 \%)$. Cette variation observée au Cameroun est aussi marquée par un taux considérable mais sur une courte période. Dans ce registre, un fait majeur est à noter pour la Guinée où la contribution de la composante qualité se situe à hauteur de $77.6 \%$ de la baisse de l'IDE. En d'autres termes, cette baisse global de l'IDE aurait été évitée rien qu'en maintenant le niveau initial de la qualité de l'éducation dans ce pays. De même, cette contribution est également négative et forte au Bénin (-59\%) et au Burundi (-42\%). Ces résultats sont logiques au regard de la baisse de la qualité de l'éducation dans ces pays. 
TABLEAU 4: Décomposition des variations de l'IDE dans différents pays selon l'approche SOS sur la période 2001-2011.

\begin{tabular}{|c|c|c|c|c|c|c|}
\hline \multirow[t]{2}{*}{ Pays } & \multirow[t]{2}{*}{ Périoded'étude } & \multirow{2}{*}{$\begin{array}{c}\text { Variation globale } \\
\text { de l'IDE }\end{array}$} & \multicolumn{4}{|c|}{ Contribution } \\
\hline & & & TNS (\%) & TAA (\%) & IEG (\%) & TS (\%) \\
\hline \multirow[t]{2}{*}{ Kazakhstan } & $2001-2011$ & 0.03875 & 0.02500 & 0.00075 & 0.00050 & 0.01250 \\
\hline & & & (65) & (2) & $(1)$ & (32) \\
\hline \multirow[t]{2}{*}{ Royaume-Uni } & $2001-2011$ & 0.01675 & -0.00075 & 0.00000 & 0.01500 & 0.00250 \\
\hline & & & $(-5)$ & (0) & (90) & (15) \\
\hline \multirow[t]{2}{*}{ Italie } & $2001-2011$ & 0.014 & -0.00025 & 0.00250 & 0.00400 & 0.00775 \\
\hline & & & $(-2)$ & (18) & (29) & (55) \\
\hline \multirow[t]{2}{*}{ Pays-Bas } & $2001-2011$ & -0.001 & 0.00150 & -0.00475 & 0.00225 & 0.00000 \\
\hline & & & (150) & $(-475)$ & (225) & (0) \\
\hline \multirow[t]{2}{*}{ France } & $2001-2011$ & $-0.0 c ̧ 035$ & -0.00200 & -0.00075 & 0.00050 & -0.00125 \\
\hline & & & $(-57)$ & $(-21)$ & (14) & $(-36)$ \\
\hline \multirow[t]{2}{*}{ Norvège } & 2001-2011 & -0.0055 & -0.00200 & -0.00450 & -0.00025 & 0.00125 \\
\hline & & & $(-36)$ & $(-82)$ & $(-5)$ & (23) \\
\hline Suède & 2001-2011 & 0.0075 & -0.00075 & -0.00125 & 0.01575 & -0.00625 \\
\hline \multirow[t]{2}{*}{ Tunisie } & 2001-2011 & 0.02775 & 0.00625 & 0.01475 & 0.00525 & 0.00150 \\
\hline & & & (23) & (53) & (19) & (5) \\
\hline \multirow[t]{2}{*}{ Cap-Vert } & 2001-2011 & 0.004 & -0.01400 & 0.02300 & -0.00325 & -0.00175 \\
\hline & & & $(-350)$ & (575) & $(-81)$ & $(-44)$ \\
\hline \multirow[t]{2}{*}{ Ghana } & $2001-2011$ & 0.1015 & 0.06025 & -0.00575 & 0.01675 & 0.03025 \\
\hline & & & (59) & $(-6)$ & (17) & (30) \\
\hline
\end{tabular}

Source: Calculs de l'auteur

Les valeurs en pourcentage représentent la part de variation de chaque composante de l'IDE sur la variation globale de l'IDE. Les autres valeurs décimales indiquent la variation de l'IDE et celle de ses composantes.

IDE, Indice de Développement de l'EPT; TNS, Taux Net de Scolarisation; TAA, Taux d'Alphabétisation des Adultes; IEG; Indice relatif au Genre; TS, Taux Survie en $5^{e}$ année.

En combinant les effets positifs et négatifs pour déterminer une contribution moyenne de l'ensemble des pays, on obtient les différentes valeurs affectées à chaque composante de l'IDE. Ainsi, par ordre décroissant d'importance on a, le taux de scolarisation (49\%), l'indice relatif au Genre (21\%), le taux d'alphabétisation (20\%) et en dernière place la qualité de l'éducation (10\%). Ces résultats sont très interpellateurs et imposent un regard attentif sur l'aspect qualitatif de l'éducation en Afrique qui contribue très peu à l'atteinte de l'objectif de scolarisation universelle.

En élargissant notre échantillon de pays, ${ }^{8}$ on trouve bien que, dans les pays proches de l'EPT et ceux ayant enregistrés des performances considérables, les contributions des composantes de l'IDE sont variables d'un pays à l'autre. Tandis que la parité fille/garçon constitue un élément capital de l'atteinte de l'EPT dans les pays à IDE élevé, l'amélioration de la qualité de l'éducation est, pour sa part, une source fondamentale de performances dans ces différents pays. Au Kazakhstan, passé de la $39^{\text {e }}$ place en 2001 à la $1^{\text {ère }}$ place en 2011, la contribution de la qualité de l'éducation est considérable (32\%). Plus encore, en Italie (18 en 2001 et $7^{\mathrm{e}}$ en 2011) l'apport de la qualité de l'éducation est plus importante, soit 55\% de l'augmentation de l'IDE. Par contre, la baisse du niveau de cette composante a conduit à la chute de la France dans le classement de l'IDE. Le bond spectaculaire du Ghana (passé de pays à très faible IDE à un pays ayant un IDE moyen), est dû principalement à la qualité de l'éducation dont la contribution se situe à hauteur de 30\% (voir tableau 4 ).

8.Considérons, sur la base des données disponibles, les 07 premiers pays dans le classement mondial de l'IDE en 2011 ainsi que les 03 premiers pays africains dans ce classement dont on peut apprécier l'évolution de l'IDE à partir de 2001.

\section{Conclusion}

Il apparait que la qualité de l'éducation compte davantage dans l'explication des différences de croissance de la productivité entre pays que les écarts en termes de nombre moyen d'années de scolarité ou de taux de scolarisation. L'évidence empirique de cette relation est nuancée lorsqu'on met en relation la qualité de l'éducation avec les OMD. La mobilisation de la technique de décomposition par l'approche SOS a donné lieu à l'appréciation de la contribution de chaque composante de l'indice de développement pour l'EPT (IDE) et particulièrement la composante « qualité de l'éducation ». Il en est ressorti que, de 1998 à 2011, la composante " qualité de l'éducation » en Afrique a contribué très peu à l'atteinte de l'objectif de scolarisation universelle. Cette faible contribution découle sans doute des politiques de massification de l'école toujours en vigueur en Afrique subsaharienne. Ces résultats sont très interpellateurs et imposent d'orienter les politiques éducatives vers les aspects qualitatifs de l'éducation avec un regard attentif sur les facteurs pouvant améliorer significativement son niveau jugé encore faible.

\section{Acknowledgements}

Nous exprimons nos remerciements à Mr Gervasio SEMEDO et $\mathrm{Mr}$ Georges KOBOU pour leurs remarques et suggestions durant la rédaction de cet article. Egalement, notre gratitude va à l'endroit aux deux lecteurs anonymes.

\section{Competing interests}

L'auteur déclare qu'elle n'a pas de relations financières ou personnelles qui peuvent l'avoir influencée de manière inappropriée en écrivant cet article. 


\section{Références}

Altinok, N.,2007, 'Essais sur la qualité de l'éducation et la croissance économique' thèse de Doctorat, Université de Bourgogne.

AuvrayC. \& Trannoy A.,1992, Décomposition par source de l'inégalité des revenus à I'aide de la Valeur Shapley, Journées de Microéconomie Appliquée, Sfax, Tunisie.

Barro, R.J.,2001, 'Education and economic growth', in J.F. Helliwell (ed.), The contribution of human and social capital to sustained economic growth and wellbeing, chapter 3 , pp. 14-41, OECD, Paris.

Baye, M.F., 2004, Growth and redistribution effects of poverty changes in Cameroon: A Shapley decomposition analysis, Forum paper, ADPR, Johannesburg, SouthAfrica.

Boccanfuso, D., Savard, L.\&Savy, B., 2009, Capital humain et croissance: évidences sur données de pays africains, GREDI, Workingpaper, 9-15, Université de Sherbrooke, Sherbrooke, Québec.

Chameni, C.N.,2006, 'A note on the decomposition of the coefficient variation squared: Comparing entropy and Dagum's methods', Economics Bulletin 4(8), 1-8.

Chameni, C.N. \&Miamo, W.C., 2012, 'Inequality of Cameroonian households: An analysis based on Shapley-shorrocksdecomposition', International Journal of Economics and Finance 4(6), 149-156.

Chantreuil, F. \&Trannoy, A., 1997, Inequality decomposition values: The trade-off between marginality and consistency, mimeo, Université de Cergy-Pointoise, Cergy-Pontoise.

Datt, G. \&Ravallion, M., 1992, 'Growth and redistribution components of changes in poverty measures: A decomposition with application to Brazil and India in the 1980s', Journal of Development Economics 38, 275-295.

Fambon, S.,2009, 'Inequality in the distribution of household expenditure in Cameroon', Annual International Conference of Development Economics, University of Frankfurt, Frankfurt.

Finnie, R.\&Meng, R.2002, 'Minorities, cognitive skills, and incomes of Canadians', Canadian Public Policy 28, 257-273.

Green, D.A.\& Riddell, W.C., 2003, 'Literacy and earnings: An investigation of the interaction of cognitive and unobserved skills in earnings generation', Labour Economics 10, 165-184.

Hanushek, E.A., 2005, 'L'importance de la qualité de l'enseignement', Finances et développement, 42(2), 15-19.

Hanushek, E.A. \&Kimko, D.D., 2000, 'Schooling, labor-force quality, and the growth of nations', American Economic Review 90(5), 1184-1208.

Hanushek E.\&Wossmann L., 2007, The role of school improvement in economic development, Working paper 12832, NBER, Massachusetts.

Kabore, T.S., 2002, The dynamics of poverty: A review of decomposition approaches and application to data from Burkina Faso, mimeo, Université de Ouagadougou, Ouagadougou.

Lazear, E.P., 2003, 'Teacher incentives',Swedish Economic Policy Review 10, 179-214.
McIntosh, S.\&Vignoles, A., 2001, 'Measuring and assessing the impact of basic skills on labor market outcomes', Oxford Economic Papers 53, 453-481.

Mulligan,C.B., 1999, 'Galton versus the human capital approach to inheritance', Journal of Political Economy 107(6 pt 2), 184-224.

Murnane, R.J., Willett, J.B., Duhaldeborde, Y. \& Tyler, J.H., 2000, 'How important are the cognitive skills of teenagers in predicting subsequent earnings?',Journal of Policy Analysis and Management19(4), 547-568.

Mussard, S.,2006, La décomposition des mesures d'inégalité en sources de revenu: l'indice de Gini et les généralisations, Cahier de recherches, Université de Sherbrooke, Sherbrooke, Québec.

Owen, G., 1977, 'Values of Games with Priori Unions', dans Heim R. et Moeschlin O. (dir), Essays in Mathematical Economics and Game Theory in honour of OSKAR MORGENSTERN, Springer Verlag, New York, 76-88.

Pritchett, L.,2001, 'Where has all the education gone?',World Bank Economic Review15, 367-391.

Rapports mondiaux de l'UNESCO sur le suivi de l'Éducation pour tous: 2002, 2005, 2006, 2007, 2008, 2009, 2010, 2011, 2012 et 2014, Paris, UNESCO.

Saha, J.C., 2004, Secteurs sociaux essentiels: goulots d'étranglement dans le processus du développement humain au Cameroun, Revue des Sciences Economiques et de Gestion, 06, N'Gaoundéré, 27-42.

Saha, J.C., 2005, Education et développement humain en Afrique de l'Ouest: des hauts et des bas. Les cas du Burkina-Faso, de la Côte d'lvoire et du Sénégal, Cornell/ CREA/INRA, conférence régionale Dakar, Dakar, 2005.

Sastre, M.\&Trannoy, A.,2002, 'Shapley inequality decomposition by factor components: Some methodological issues', in M.P. Seidl C. \& A.F.Shorrocks (éds.), Journal of Economics 9(Suppl), 51-90.

Shapley, L., 1953, 'A value for n-Person games', in H. W. Kuhn \& A.W. Tucker (eds.), Contributions to the Theory of Games, vol. 2, Princeton University Press, New Jersey.

Shorrocks, A.F., 1999, Decomposition procedures for distributional analysis: A unified framework based on Shapley value, Department of Economics, University of Essex, Colchester, UK, 1-38.

Shorrocks, A.F. \&Kolenikov, S., 2001, Poverty trends in Russia during the transition mimeo, WIDER and University of North Carolina, Discussion paper UNU-WIDER, Helsinki.

Silber, J., 2004, 'Inequalities: Theory, experiments and applications', European Journa of PoliticalEconomy 20, 813-820.

UNESCO (1990), Déclaration mondiale sur l'éducation pour tous et le Cadre d'action pour répondre aux besoins éducatifs fondamentaux. Conference mondiale sur l'éducation pour tous, Jomtien , Thaïlande, 5-9 mars 1990, Paris, UNESCO.

UNESCO (2000), Cadre d'action de Dakar : L'Éducation pour tous : tenir nos engagements collectifs. Forum mondial sur l'éducation Dakar, Sénégal 26-28 avril 2000. Paris, UNESCO.

Woessmann, L., 2002, 'Schooling and the quality of human capital', Journal of Political Economy 70(5), 945-971. 